\title{
In Situ LC-TEM Studies of Corrosion of Metal Thin Films in Aqueous Solutions
}

Jeung Hun Park ${ }^{1,2}$, See Wee Chee ${ }^{3}$, Suneel Kodambaka ${ }^{1}$, and Frances M. Ross ${ }^{2}$

${ }^{1}$ Department of Materials Science and Engineering, University of California Los Angeles, 410 Westwood Plaza, Los Angeles, CA 90095, USA

${ }^{2}$ IBM T. J. Watson Research Center, 1101 Kitchawan Road, Yorktown Heights, NY 10598, USA

${ }^{3}$ Centre of Bioimaging Sciences, Department of Biological Sciences, National University of Singapore, 14 Science Drive 4, Singapore 117557

Chemical reactions with the environment result in corrosion or degradation of the properties of materials [1]. Metals in contact with aqueous solutions, depending on their surface morphology, undergo different modes of corrosion [2]: general, pitting, crevice, inter-granular, galvanic, and erosion corrosion, environmentally induced fracture, and dealloying. In spite of its importance, many aspects of localized corrosion of metals are not well understood [3], probably because it is often difficult to monitor the corrosion processes at high spatial resolution under a liquid layer. Liquid cell transmission electron microscopy (LC-TEM), coupled with electrochemical control and analytic functions, enables the observation of structural changes and chemical processes in liquid phases. It provides a combination of temporal and spatial resolution that is difficult to achieve using other characterization techniques [4], and has yielded detailed information on corrosion kinetics in liquid solutions $[3,4]$.

In this work, we studied corrosion of metal films in aqueous solutions using in situ LC-TEM. We examined general, pitting, and galvanic corrosion in several metals ( $\mathrm{Au}, \mathrm{Al}, \mathrm{Ni}, \mathrm{Cu}$, and $\mathrm{Zn}$ ) under different aqueous solutions. The samples are 20 - $50 \mathrm{~nm}$ thick metal films deposited by electron beam evaporation over a $50 \mathrm{~nm}$ thick silicon nitride membrane of liquid cell window chips. The experiments were carried out in a FEI CM30 TEM, operated at $300 \mathrm{kV}$, using a continuous flow Hummingbird Scientific LC-TEM holder. Deionized water was first introduced into the liquid cell to completely remove air bubbles inside the cell. Then the liquid, either deionized water or a solution containing $\mathrm{HCl}$, $\mathrm{H}_{2} \mathrm{SO}_{4}, \mathrm{CuSO}_{4}, \mathrm{PbSO}_{4}, \mathrm{NaCl}, \mathrm{ZnCl}_{2}$, and $\mathrm{ZnSO}_{4}$, was introduced. We used low dose imaging protocol and verified that these solutions can be examined for long periods (many hours) without visible beam effects in the liquid. Figure 1 presents corrosion morphologies of a Ni film in deionized water $(\mathrm{pH}=7)$. This corrosion process took place under a constant electron beam illumination and a flow rate of 5 $\mu \mathrm{l} / \mathrm{min}$. Bright field TEM images showed that dissolution of Ni films occurs via the general corrosion mode in which chemical or electrochemical reactions proceed over the entire exposed surface at approximately the same rate of dissolution. The electron beam accelerates the process, presumably via reactive species created by beam-induced radiolysis of water [5,6]. Figure 2 shows corrosion morphologies of an Au film in $0.1 \mathrm{M} \mathrm{HCl}(\mathrm{pH}=1.26)$. This corrosion took place during linear sweep voltammetry. Image analysis showed that the corrosion of Au films occurs via dissolution of individual grains.

We will describe how measurements of the local dissolution kinetics - morphology, composition, and the etching rate of these metal films as a function of solution composition, $\mathrm{pH}$, and metal thickness provide insights into corrosion processes with good spatial and temporal resolutions.

\section{References:}

[1] B. A. Shaw and R. G. Kelly, The Electrochemical Society Interface Spring (2006) 24.

[2] H.-H. Strehblow et al. in "Corrosion Mechanisms in Theory and Practice (3rd Ed., 2012)", ed. P. Marcus, (CRC Press , New York) 1 - 572. 
[3] S. W. Chee et al., Chemical Communications 51 (2015) 168.

[4] N. de Jonge and F. M. Ross, Nature Nanotechnology 6 (2011) 695.

[5] J. H. Park et al., Microscopy and Microanalysis 19 Suppl. 2 (2012) 1098.

[6] J. H. Park et al., Microscopy and Microanalysis 18 Suppl. 2 (2013) 486.

[7] We gratefully acknowledge funding from the National Science Foundation (NSF-GOALI: DMR1310639), and technical assistance of M. C. Reuter and A. W. Ellis.
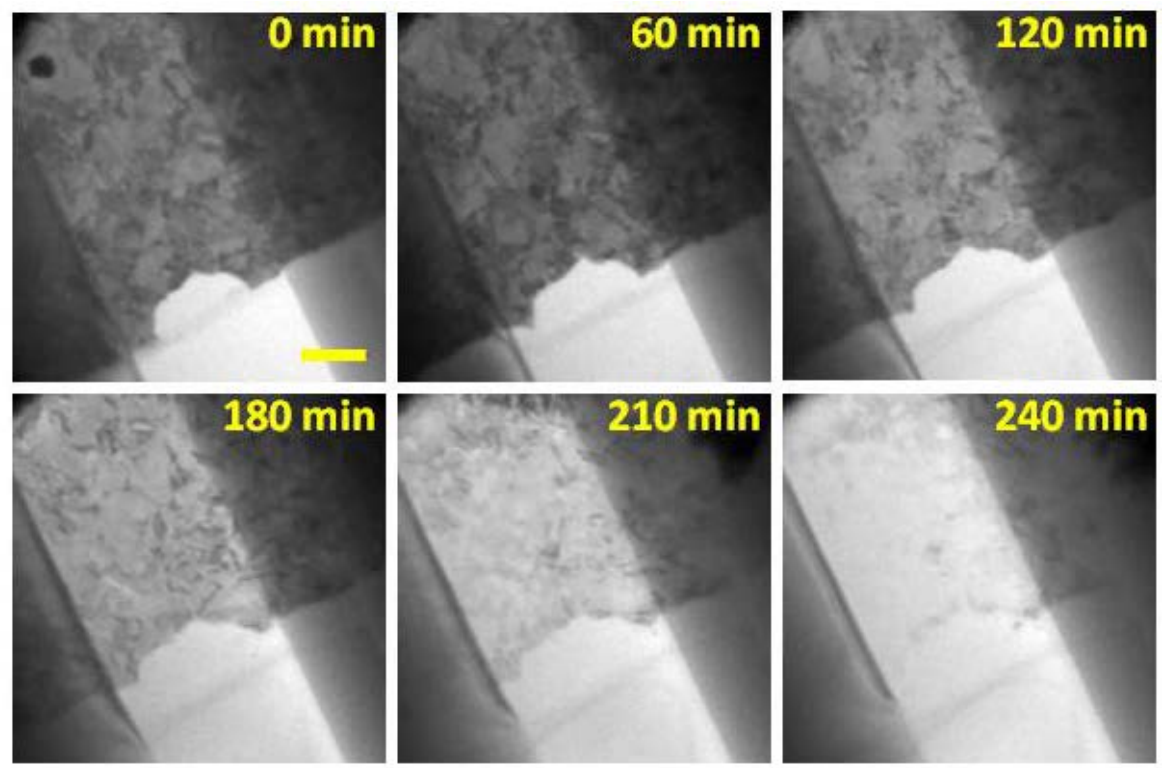

Figure 1. Dissolution of a Ni film: Images extracted from a bright field TEM movie recorded while flowing deionized water at a rate of 5 $\mu \mathrm{l} / \mathrm{min}$. Scale bar is $500 \mathrm{~nm}$.
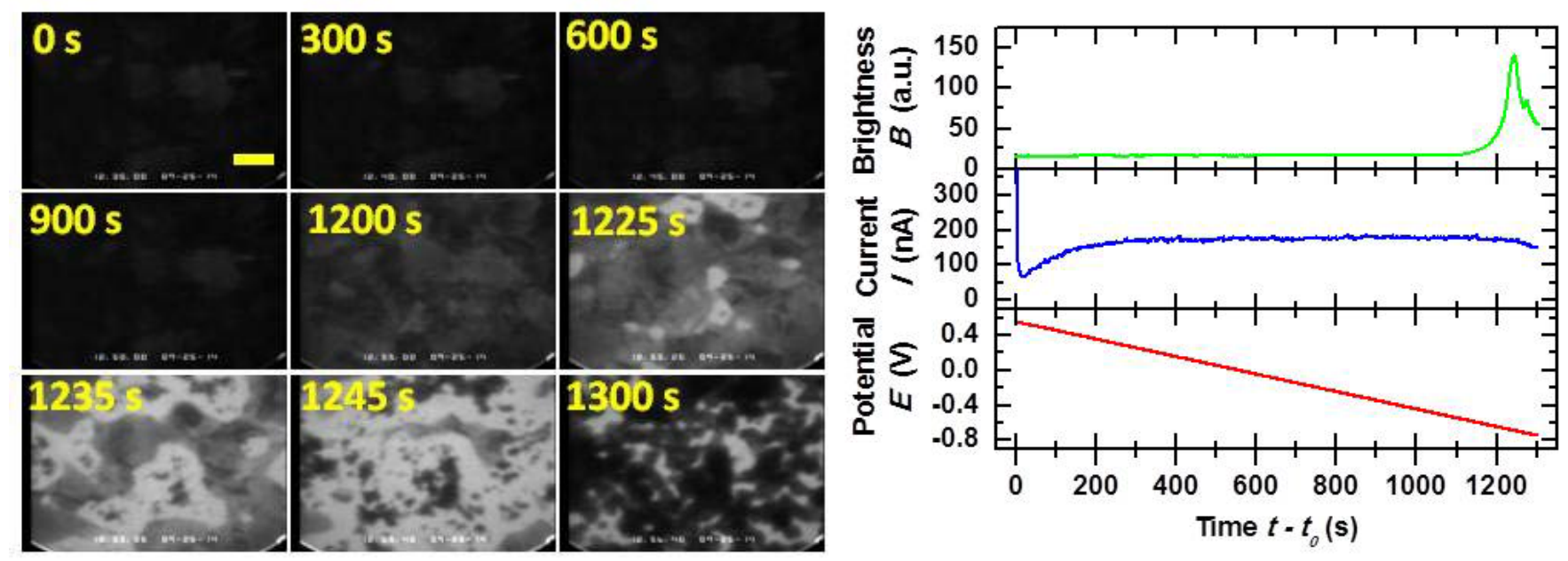

Figure 2. Dissolution of an Au film on silicon nitride under applied voltage: (Left) Images extracted from a bright field TEM movie obtained during electrochemical etching of Au film in $0.1 \mathrm{M} \mathrm{HCl}$ solution. In the final two images, beam-induced redeposition of Au is visible as darker features in bare regions of the silicon nitride window. Scale bar is $200 \mathrm{~nm}$. (Right) Linear sweep voltammetry data are recorded simultaneously. The voltage sweeps from $+0.35 \mathrm{~V}$ to $-0.8 \mathrm{~V}$ at $1 \mathrm{mV} / \mathrm{s}$. Current recorded over the whole electrode is also shown. The top graph shows the average image brightness, indicating the approximate $\mathrm{Au}$ thickness and onset of dissolution in the field of view. 